\title{
FLT3-Internal Tandem Duplication High Allelic Ratio
}

National Cancer Institute

\section{Source}

National Cancer Institute. FLT3-Internal Tandem Duplication High Allelic Ratio. NCI

Thesaurus. Code C123406.

A molecular genetic finding in acute myeloid leukemias indicating that FLT3-internal tandem duplication (FLT3-ITD) is the dominant lesion, and which is present in the majority or all of the leukemic cells. 